\title{
Aborder la culture française à travers la chanson ${ }^{1}$
}

\section{(Abordar la culturafrancesa mediantelacanción)}

\section{Virginia Boza Araya ${ }^{2}$}

Universidad Nacional, Costa Rica

\section{résumé}

La chanson a toujours été une expression profondément populaire, ancrée dans les cultures et les traditions des peuples. Elle suit l'évolution de la société et des individus en évoquant autant leurs joies, leurs malaises que tous les problèmes qu'ils affrontent au quotidien. Dans le domaine de la didactique du Français langue étrangère, la chanson offre aux enseignants la possibilité de diversifier, d'élargir les approches et les applications sociolinguistiques dans la classe. Dans cet article on fait un bref rappel des approches pédagogiques concernant la didactique de la culture et on propose une stratégie pédagogique originale.

\section{resumen}

La canción es expresión popular ligada a las culturas y a las tradiciones de las comunidades. Suele acompañar la evolución de la sociedad evocando sus valores y emociones y demás hechos de su vida cotidiana. En el campo de la didáctica del francés como lengua extranjera, la canción ofrece la posibilidad de diversificar y ampliar las perspectivas y las aplicaciones sociolingüísticas en clase. En este artículo se describen los enfoques aplicados a la enseñanza de la cultura y se propone una estrategia pedagógica original.

Mots clés : chanson populaire, culture, Français langue étrangère, société française.

1 Recibido: 23 de febrero de 2012; aceptado: 19 de febrero de 2013.

2 Escuela de Literatura y Ciencias del Lenguaje. Correo electrónico: dantrou @ hotmail.com.

$$
L_{\text {etras }} 53 \text { (2013), ISSN 1409-424X; EISSN 2215-4094 }
$$


Palabras clave: canción popular, cultura, Francés como lengua extranjera, sociedad francesa

Peut-on vraiment enseigner quelque chose qui serait à l'opposé de ce que pense, de ce que vit, de ce que pratique depuis si longtemps celui à qui l'on s'adresse ? s'interroge Pierre Dumont (1998 : 127). En effet, celui qui a déjà dû vivre dans une autre société que la sienne, avec en outre une culture différente et parfois même opposée, connaît la difficulté de pouvoir la comprendre, l'acquérir, l'assimiler et vivre en fonction de ses codes de conduite. Sa compréhension et son acquisition sont d'autant plus difficiles lorsqu'on se trouve dans un pays non francophone comme le Costa Rica qui n'a aucun contact avec la ou les cultures étudiées. Dans ce contexte, comment l'aborder ? Quelles approches pédagogiques privilégier? Quelle stratégie pédagogique faut-il mettre en place ? Quels outils sont les plus performants?

Afin de mieux faire appréhender la didactique de la culture, il est essentiel de faire un rappel des différentes approches mises en pratique par les didacticiens soit séparément soit combinées. L'enseignement de la culture peut donc être envisagé du point de vue représentatif. Le moyen privilégié par cette approche est la littérature qui rend plus accessible les réalités recréées par les artistes. Dans ce sens toute œuvre artistique rend possible la découverte des sentiments, des idéaux, des vécus d'une société. Parmi ces créations celle qui se rapproche le plus de la littérature est la chanson dont les paroles peuvent être étudiées comme un poème et son contenu est riche en éléments ethno-socioculturels. C'est cet aspect que cette étude essaie de mettre en évidence car la chanson peut être considérée comme une passerelle entre la langue et la culture. Elle constitue en outre une ouverture vers d'autres moyens d'expression : musique, littérature, cinéma et aussi vers d'autres domaines d'études : sociologie, anthropologie, sciences sociales, etc. 
La deuxième approche se penche plutôt sur les faits qui déterminent les Français au niveau spatio-temporel à savoir la géographie et l'histoire qui marquent l'évolution de cette société, qui peuvent expliquer certains fondements tels que les Droits de l'homme et l'humanisme, entre autres.

Une autre manière de découvrir une culture étrangère consiste en une série de repères culturels qui permettraient, tel un puzzle, d'établir une vision d'ensemble de la société.

Contrairement aux approches précédentes (définies par rapport aux contenus), l'approche qui pourrait être définie comme « itinéraire d'apprentissage » est déterminée par ce qui est appris par chaque étudiant. Autant les premières requièrent un programme définit au préalable, cette dernière n'a pas de directives particulières et se construit au gré de l'étudiant.

L'approche prédominante actuellement est celle qui vise à favoriser les échanges entre les cultures, l'ouverture vers l'autre et donc une plus grande tolérance. C'est justement à ce niveau que l'étude de la chanson peut être un atout car elle permet de sensibiliser l'élève au respect de l'autre, au respect de sa propre culture, aspect qu'il faut privilégier en ces temps d'intolérance et de racisme soit dans un milieu multiculturel soit à l'intérieur d'une culture donnée. Une meilleure connaissance des cultures de différents pays permet d'affirmer sa dissemblance. Ceci contribue à réaffirmer ses acquis et, par la même occasion, à comprendre le phénomène de l'altérité (de la différence, de la diversité) propre à une société. Cette altérité est d'autant plus évidente dans une société multiculturelle comme celle de la France qui compte une grande diversité d'immigrés. Venant d'horizons différents, avec des codes et des perceptions autres, comment leur imposer ceux de la culture d'accueil. Doit-on, peut-on effacer la culture d'origine et la remplacer par une autre que ce soit du point de vue pragmatique ou éthique ? Les enfants des immigrés, eux, vivent dans l'altérité totale car ils sont partagés entre deux mondes distincts : celui de leurs parents et celui de la société dans laquelle ils évoluent. En fait, 
leur double appartenance est difficile à vivre car ils ne s'identifient à aucune des deux sociétés ce qui cause souvent de problèmes d'intégration. Cette difficulté ajoute une autre dimension à l'altérité de la société. Cette approche est orientée vers un savoir-faire plutôt que vers un savoir (contenus) puisqu'il faut savoir agir dans divers milieux. Cette vision s'est évidemment élargie à l'enseignement des langues poussé par les échanges dans la propre société tant sociaux que commerciaux et culturels de plus en plus fréquents entre les divers pays du monde. Donc le mot d'ordre est « Ouverture».

Une autre perspective envisagée dans l'actualité est la compétence ethno-socio-culturelle définie par Henry Boyer (De la compétence ethno-socioculturelle - 1995 : 43) comme « un ensemble composite de traits constitutifs d'imaginaires collectifs » et qui est en interaction permanente avec une compétence discursive textuelle (savoir d'ordre langagier) et une compétence référentielle (savoir-faire). Ces représentations collectives correspondent, selon monsieur Boyer, à des « images », des « valeurs », des « attitudes », des « mentalités », des « croyances », des « visions du monde », des « opinions », des « évaluations », des « préjugés », des «mythes », des « idéologies », des « stéréotypes », etc. Ces imaginaires présentent divers degrés de stabilité ou de permanence car, étant inhérents à l'être humain, ils changent ou évoluent avec la société elle-même. Certaines représentations se rapportent au vécu des individus comme, par exemple, l'argent et le travail qui sont très liés entre eux et qui ont des incidences dans le cœur même de la famille et de la société. Ils génèrent une série de comportements, de sentiments, d'attitudes, de préjugés partagés par la majorité des individus de celle-ci et qui leur sont propres.

Henri Boyer, pour sa part, considère que d'autres imaginaires sont plus instables puisqu'ils correspondent à un consensus soit d'une partie ou de la majorité de la société à un moment donné de son histoire. Citons par exemple des principes tels que les Droits de l'homme inexistants dans les sociétés occidentales jusqu'au xviiì siècle. Ils ont été adoptés comme principes inviolables par bon nombre d'individus 
et de sociétés après la Révolution française et restent d'actualité particulièrement dans la société française. Ils constituent d'ailleurs un « trait d'identité collective ». La tolérance a particulièrement été mise à l'ordre du jour dans les années 80 avec le slogan «Ne touche pas à mon pote » comme une réaction contre le racisme qui s'imposait de plus en plus dans la société française. D'autres valeurs, ayant cours à une certaine époque, sont les idéologies politiques. Un très bon exemple est celui du socialisme dans les années 80/90 auxquelles adhèrent de moins en moins les citoyens français qui se tournent plutôt vers les valeurs capitalistes. Parfois celles-ci découlent d'un sentiment de révolte collective contre une mauvaise politique ou des peurs éprouvées par les Français comme en témoigne la poussée de l'extrême droite avec le Front National et son slogan « La France aux Français ». Parfois ces tendances détournent, à leur profit, des emblèmes patrimoniaux tels que l'image de Jeanne d'Arc que ce groupe politique s'est approprié pour essayer d'attirer les personnes qui s'y reconnaissent. La valeur emblématique de Jeanne d'Arc contient certains implicites. Rappelons que cette héroïne a chassé les envahisseurs anglais. Aussi, il semblerait évident qu'associée à ce mouvement extrémiste le message diffusé dans l'actualité française serait celui de chasser les immigrés et donc une incitation au racisme. Mais ce n'est qu'une hypothèse parmi tant d'autres. Elles peuvent également correspondre à une conception particulière de la vie. C'est le cas du mouvement Hip-Hop apparu en France à la fin des années 80 et qui est propre à une partie de la société issue des milieux défavorisés. Ces phénomènes culturels étant indissociables de la langue créent à leur tour des sociolectes (termes spécifiques à une société donnée ou à une partie de celle-ci) qui seront à la mode aussi longtemps que le mouvement persiste et qui fonctionnent comme des «marqueurs d'identités » puisqu'ils identifient les membres d'un groupe social ou professionnel. Tel est le cas du parler des jeunes rappeurs qui est en vogue dans les cités. Un autre exemple de marqueurs d'identité, par rapport aux Français, est le mot « Hexagone » utilisé à la place 
du mot France. Il est synonyme de patrie, de nation. Certains sont assez récents. C'est le cas des termes « juilletiste » et « aoûtien » qui désignent les personnes qui prennent leurs vacances soit en juillet soit en août. Ainsi définis par Henry Boyer, ces « imaginaires collectifs » s'accordent plus à la définition de culture véhiculée par les Sciences sociales avec laquelle les professeurs de français ne sont pas familiarisés. De ce fait, il n'est pas facile de les introduire dans la classe F.L.E. ou dans un cours de « civilisation ».

Plusieurs expériences pédagogiques avec des documents authentiques (publicités, vidéos, documents sonores divers entre autres) ont été mises en pratique pour essayer de répondre à cette interrogation. Pour transmettre cette connaissance la chanson a été choisie par l'auteur de l'étude : «La chanson, miroir d'une societe : support pedagogique dans un cours de civilisation français » parce qu'elle est non seulement une source inépuisable d'informations sur la société mais aussi un outil pédagogique très utile pour faire connaître la culture et développer les compétences linguistiques. Ainsi que l'affirme le chanteur - compositeur Yves Duteil «La chanson, c'est quelque chose qui nous suit du berceau jusqu'au tombeau. Les chansons, c'est nous, c'est notre histoire. Ça nous raconte » $(1995$ : 52). Ces affirmations mettent donc l'accent sur l'aspect socio-linguistique et culturel de la chanson qui est le reflet de l'individu et l'expression des traits civilisationnels qui marquent son être et par cela même de la société dans laquelle il vit. En outre, la chanson constitue un excellent déclencheur de l'oral qui permet d'élargir la compétence communicative.

On peut, par exemple, choisir d'aborder la France avec ses grandes villes et régions en mettant en relief la rivalité existante entre Paris et les régions et l'image de la société française. Pour donner une image de Paris et de la vie parisienne on peut s'intéresser aux monuments tels que : La tour Eiffel, L'Etoile, l'Arc du Triomphe, l'Obélisque, Montmartre, Le Sacré Cœur, La cathédrale de Notre Dame entre autres. On peut les découvrir grâce à des chansons telles Il est 5 heures, Paris se lève de Jacques Dutronc (1968) 
car elle offre une description assez fidèle de Paris (ses habitants, ses monuments, son activité). C'est l'image traditionnelle, touristique d'hier et d'aujourd'hui, le " Paris lumière », le Paris paillette des fêtes et des cabarets tels que Le Moulin Rouge et les Folies Bergères. La chanson inclut la plupart des stéréotypes existant sur Paris. On peut aussi faire découvrir deux tendances quant à la représentation de Paris. L'une souriante, celle du Paris ensoleillé où les gens ont retrouvé leur bonne humeur ainsi que l'exprime Alain Souchon dans «Les gens sont devenus gentils » (T'aurais dî venir - 1974). Il semblerait que les gens y sont toujours en vacances car il y a de la musique partout. Paris ressemble à une fourmilière. L'animation y est permanente. Les travailleurs dans la vie quotidienne tels que les boulangers, les balayeurs, les ouvriers des usines, les éditeurs et les vendeurs de journaux se pressent, les employés prennent le RER et le métro pour aller travailler. L'autre image de Paris est moins joyeuse, à Paris plein de vie s'oppose la blancheur morne et la grisaille de l'hiver, les places vides, inanimées. On peut l'analyser avec des chansons telles que Neige de Gilbert Lafaille -1977, Gare de Lyon de Barbara -1970, Petite annonce d'Alain Souchon (1974). En effet, Tout est statique «Le temps se fige, plus rien ne bouge » entre autres. D'autres chansons s'attardent sur les cafés tels que le Flore (Paris, le Flore, Etienne Daho - 1980) et le Paris romantique des jardins publics, des parcs aux amoureux (Au parc Monceau, Yves Duteil (1981) les cabarets et la vie nocturne. Les chanteurs expriment aussi leur attachement à leur ville. On peut étudier non seulement l'Hexagone avec ses différentes régions : la campagne, les villes et les banlieues mais aussi la France multiculturelle avec ses Français d'origine européenne ou ceux d'outre-mer, d'origine arabe, africaine et bien d'autres ; la situation sociale et économique de cette population; ses sentiments et ses actions ; les aspects culturels et historiques tels que La langue française, la musique, la littérature, l'histoire : période, événement, personnages ; l'histoire Coloniale : pays, situation coloniale et attitude coloniale, entre autres. Si le professeur 
décide de sensibiliser les étudiants à l'étude de la littérature en l'introduisant par une chanson, il pourrait utiliser la chanson Emma (Je m'appelle Emma) de Nicole Croisille (1999) pour Madame Bovary, ou Les Misérables de Patricia Kaas, ou bien des chansons ${ }^{3}$ consacrées au Petit Prince voire la comédie musicale ${ }^{4}$. Un prolongement possible serait l'écoute d'un extrait du livre et /ou regarder le film et analyser avec les élèves soit le document sonore soit la mise en scène pour connaître le degré du respect du texte. Dans l'actualité, la chanson apparaît comme un outil intéressant pour approcher la littérature autrement. D'ailleurs, depuis 1999, l'un des genres musicaux en vogue est la comédie musicale qui s'inspire particulièrement de grands classiques de la littérature universelle : Notre Dame de Paris (1997), Les Misérables (1989), Roméo et Juliette (1999), Le roi soleil (2000), Gladiateur (2000), Le petit Prince (2002), Cendrillon (2002), entre autres. Ces comédies, de par leur musique, l'adaptation de l'œuvre et le cadre féerique, fascinent le public et ont le mérite de rendre accessible l'œuvre de ces grands écrivains motivant un plus large public et les élèves en particulier à lire le roman comme un plaisir supplémentaire. Le visionnement du spectacle en vidéo pourrait les stimuler pour monter leur propre adaptation. Cette simulation pourrait être envisagée comme un projet de classe qui permettrait l'étude des différentes chansons en groupe avec l'explication du texte, les références par rapport au livre, l'histoire racontée, les événements sociaux illustrés par l'écrivain, sans oublier la biographie de celui-ci. Ce travail permettrait en outre d'introduire quelques notions sur l'histoire de la France en les rendant plus accessibles que par le biais d'une classe magistrale. C'est un travail effectué par les élèves pour les élèves qui demande des allées/venues dans le temps et dans l'espace. Le rôle du professeur se résume au rôle de simple conseiller ou de coordinateur de l'activité valorisant ainsi les apports des étudiants et favorisant, par la même occasion, l'apprentissage.

\footnotetext{
3 Gilbert Bécaud, Le petit prince est revenu; Mylène Farmer, Dessine-moi un mouton, 1999.

4 Elizabeth Anaïs, Richard Cocciante, Le petit Prince, 2002.
} 
Rappelons qu'il existe des clips vidéo sur les chansons clés des comédies qui font la promotion de celles-ci à TV5. Ils se sont avérés très utiles dans le cadre de l'apprentissage du FLE à la Universidad Nacional, au Costa Rica. En effet des étudiants qui ont eu l'occasion de visionner les clips de Belle et Le temps des cathédrales de la comédie Notre Dame de Paris et On dit dans la rue, Les rois du monde de Roméo et Juliette adoraient ces titres au point de les connaître par cour. Cela a permis de les étudier en classe avec d'excellents résultats en ce qui concerne la participation des étudiants et l'enthousiasme manifesté pour cette activité. Rappelons que la littérature en chanson ne se limite pas à la prose car nombreux poèmes sont mis en musique comme par exemple Le pont Mirabeau qui vient récemment d'être chanté par Marc Lavoine, ou encore la compilation Brassens, Gréco, Montand et Mouloudji chantent les poètes éditée par Hachette en collaboration avec le ministère français de Relations extérieures en $1982^{5}$. Eventuellement le professeur peut profiter de l'occasion, si besoin est, pour faire un exercice de phonétique.

La stratégie proposée dans cet article privilégie la perspective ethno-socioculturel qui permet d'appréhender les principales particularités des sociétés et la perspective interculturelle qui, au moyen de la comparaison avec la propre culture, met en relief celle de la langue seconde et sensibilise à l'altérité des cultures étudiées tout en s'appuyant sur les contenus linguistiques des textes étudiés.

Les objectifs de cette démarche sont les suivants :

- Aborder différents aspects des cultures francophones

- $\quad$ Faire découvrir leur diversité culturelle (habitudes, mœurs, valeurs, vie quotidienne).

- Développer la perception des faits sociaux, des représentations, des stéréotypes, des emblèmes des cultures.

5 Jean-Claude Demari, « De « Gentille alouette » à « Lambé An Dro »- 50 ans de chanson en classe FLE ». Le Français dans le Monde 318 (1999) : 52-55. 
- Sensibiliser à l'altérité de cette société française vis-à-vis de la culture de l'apprenant

- Développer l'appréciation de la chanson francophone : paroles, rythmes, messages

- Donner des informations et faire des mises à jour sur la société qui permettent d'aborder différents sujets lors de discussions avec des francophones.

- $\quad$ Apprendre des codes de comportements pour se débrouiller dans certaines situations sans choquer l'interlocuteur.

- Développer la compétence communicative en favorisant l'apprentissage linguistique : étude du vocabulaire dans son évolution (des sociolectes, des interlectes, des figures de style). Rappelons que la chanson est un hybride de la prose et du poème. Elle raconte des anecdotes, des histoires, des scènes ou des situations sous forme de poème et donc, comme celui-ci, elle est écrite en vers, elle contient des rimes, des figures de style et des images.

La démarche proposée se veut interactive et dynamique car l'apprenant est toujours amené à exprimer ses opinions, ses perceptions des différentes situations présentées, ses connaissances, ses intuitions. C'est lui qui doit élucider, dès le départ, les informations, les messages transmis par la chanson. Il doit essayer de deviner, de faire des hypothèses concernant les aspects sociologiques exposés dans les chansons.

\section{1) Phase de sensibilisation}

Il est important d'entamer cette phase en demandant aux élèves d'établir une liste d'une dizaine de mots ou d'exprimer des idées concernant le thème qui sera étudié ou présenté par la chanson ce qui permettra d'évaluer la perception des apprenants par rapport à un sujet donné. Cette première activité de sensibilisation sera, bien entendu, choisie en fonction du niveau de la classe et du public. Ce 
premier contact peut également se faire à partir d'images apportées par les apprenants qui seront commentées dans le cours et classées selon les notions qu'elles mettent en valeur. Ainsi, par exemple, si le professeur choisit d'analyser la vie parisienne et les sentiments que les Parisiens éprouvent pour leur ville, il peut demander à ses étudiants soit d'apporter des cartes postales ou des photos de monuments, de quartiers, d'endroits connus de Paris en pleine activité ou bien tout simplement de proposer des mots qui, à leur avis, dépeignent le mieux Paris.

\section{2) Phase de découverte de la chanson}

La première écoute se fera sans aucun élément distrayant de façon à concentrer l'attention des apprenants sur la compréhension orale de la chanson. Cette écoute sans texte suscitera beaucoup de commentaires puisqu'il est fait appel à la capacité de compréhension et de perception des étudiants.

Les premières questions seront posées pour mettre en relief la qualité musicale et les sentiments des élèves concernant les paroles, la musique, l'interprétation, la voix du chanteur et l'impression crée par l'ensemble de ces éléments chez eux. Une large part sera accordée à cette étape pour tirer un maximum de profit des échanges d'informations entre les étudiants. Ce travail favorisera, en même temps, l'expression orale tout en mettant en confiance les élèves.

La deuxième écoute se centrera sur le contenu de la chanson et donc sur ce que les apprenants comprennent. Le professeur notera au tableau les mots et/ou les notions énoncées par les élèves et demandera à ceux-ci d'expliquer ce que ces mots évoquent pour eux.

La troisième écoute se fera avec un support image, sorte de clip qui explicitera les informations contenues dans le texte et qui permettra de vérifier les hypothèses avancées par les étudiants. L'enseignant profitera de ce support pour l'analyser avec les apprenants de façon à essayer de compléter les informations apportées par la chanson. 
3) Phase d'explication des contenus culturels et linguistiques

Tout d'abord, le professeur demandera aux apprenants de présenter le contexte (la situation, le portrait, la description...) ébauchées par le texte selon le thème étudié. Après la reprise des contenus, il posera des questions sur les faits présentés. Il notera au tableau les réponses des étudiants et les analysera avec eux afin d'expliciter les situations évoquées. Puis il expliquera le vocabulaire, en particulier si la chanson a un vocabulaire populaire ou argotique. Il faut éviter au maximum de traduire en langue maternelle. Pour expliquer ou définir certains termes le professeur peut avoir recours à des synonymes ou des mots transparents. Cependant, devant un terme difficile la traduction n'est pas exclue à condition que l'expression traduite soit plus parlante et bien entendu dans la logique du document. Il est important d'être attentif à ce que les étudiants n'aient pas une perception erronée des réalités françaises étudiées, le professeur doit donc bien guider leur réflexion et corriger l'orientation s'il en ait besoin.

\section{4) Phase d'acquisition}

Dans cette phase les étudiants auront une vision d'ensemble de la chanson et ceci grâce au Karaoké spécialement conçu par le professeur en associant les images et les textes. C'est le moment de la découverte des paroles. C'est aussi une phase où une large place sera accordée au plaisir de chanter. La chanson peut servir ainsi de point de départ pour l'étude de la culture française. La chanson est donc un déclencheur culturel qui facilite l'accès à la civilisation d'une manière ludique et motivante. De plus chaque sujet abordé pourra être illustré par d'autres documents authentiques tels que des interviews diverses, des articles de journaux, des reportages vidéos, des documents sonores, des extraits littéraires. 


\section{5) Phase d'application}

Fidèle à l'objectif de rendre le cours plus dynamique et interactif possible, cette phase, très liée à la phase interculturelle, sera centrée sur la créativité et la participation des élèves. Elle peut aller d'un simple jeu de rôles à une simulation globale en passant par l'écriture d'une nouvelle chanson soit sur le même registre soit sur un autre registre, en respectant le champ sémantique ou bien un sujet contraire à celui de la chanson comme par exemple : l'amour / la haine, la violence / la paix, Paris / Province, les Français / les immigrés.

D'autres possibilités d'application de cette démarche pourraient exiger un travail plus actif des étudiants qui pourraient faire une recherche en groupe pour analyser et compléter les informations sur un thème donné. Il constituerait un module qui aurait besoin de séances de préparation et d'exploitation mais, par la participation active et valorisante des étudiants, pourrait s'avérer très motivante et formatrice. Ce type d'activité permettrait ainsi d'étudier plusieurs chansons sur un même sujet qui donnerait une image d'ensemble. Par exemple, si l'enseignant souhaite faire connaître l'Hexagone sur divers aspects, il pourrait diviser les étudiants en groupes, donner à chaque équipe une chanson consacrée à une région française. Les élèves commenceront par analyser le texte, puis compléteront une fiche (Annexe 1) où ils noteront des informations générales : Nom de la région, localisation, description géographique, climats, nombre d'habitants, villes principales, activité économique, sites touristiques, spécialités gastronomiques, folklore, bref rappel historique, entre autres. Chaque groupe fera écouter sa chanson en classe, expliquera son contenu culturel et linguistique et exposera les caractéristiques de sa région en les accompagnant d'affiches, des photos, des vidéos pour donner l'image la plus complète possible. Bien entendu, ce travail suppose que les élèves sont déjà familiarisés avec l'analyse de la chanson et ont une certaine autonomie. Ainsi lorsque chaque équipe aura présenté son travail, il sera possible de les comparer, d'en discuter pour se forger une image de la diversité, éventuellement de voir les similitudes afin d'établir 
leur francité. Cette mise en commun serait l'occasion d'élaborer une synthèse écrite qui mettrait en relief les multiples acquisitions aussi bien au niveau linguistique que culturel. D'autre part ce travail, témoignerait de l'effort de compréhension au sens le plus large.

En ce qui concerne l'évaluation de l'acquisition des connaissances, il serait intéressant de faire préparer un jeu de «Questions pour un champion« qui permettrait non seulement de vérifier les connaissances tout en jouant, mais aussi de garder ainsi le caractère ludique et donc le plaisir d'apprendre en valorisant, par la même occasion, le travail et les capacités des élèves. Cette activité pourrait démarrer par le visionnement de l'émission à la télévision. Après l'analyse du déroulement de l'émission, chaque équipe rédigera les questions et les réponses. Ensuite les étudiants s'affronteront dans la simulation du jeu et s'élimineront les uns aux autres. Les trois élèves gagnants auront un prix. Cette étape pourrait être enregistrée. Outre cette activité ludique et éducative le professeur pourra organiser un débat sur l'un des sujets ébauchés par l'étude des régions qui plongent les apprenants dans l'actualité française par exemple, les revendications indépendantistes de certaines régions (La Corse, Les Antilles, La Bretagne, entre autres). C'est un fait qui bouleverse la France. Immanquablement, il faudra faire un bref rappel de l'histoire de France et de l'histoire coloniale afin que les apprenants comprennent les enjeux d'une telle démarche. Les différents problèmes pourront être illustrés avec des articles ou des reportages transmis dans les journaux télévisés (tel est le cas de la Corse) qui défraie la chronique depuis quelques années. D'ailleurs, en ce qui concerne les Bretons, le groupe qui illustre le mieux cette scission de la France est le groupe musical Manau qui est dans la lignée d'Alain Stivell.

\section{6) Phase interculturelle}

Une fois la chanson analysée, du point de vue civilisationnel et linguistique, il serait souhaitable (afin de mieux faire comprendre la particularité de la culture française où de la «Francité » telle que l'a 
expliqué Michel Boiron) de comparer les valeurs, les emblèmes, les usages, les attitudes de la culture des apprenants. Ils seront amenés à commenter les différents faits de société en expliquant quelles sont leurs opinions où quelles attitudes adopteraient-ils dans la même situation. Différentes activités peuvent être réalisées où le vécu des élèves sera mis en pratique. L'expression orale sera favorisée car elle permet l'interaction des étudiants et donc une meilleure compréhension des faits grâce aux apports de chacun. Certaines de ces activités doivent être très créatives et ludiques : jeu de rôles, pièces de théâtre, simulations globales, chanson traitant le même thème mais exprimant la perception ou les croyances des apprenants. Évidemment cela dépendra du niveau de connaissances linguistiques des étudiants. Ainsi par exemple la discussion sur les revendications indépendantistes pourrait lancer un débat sur le même sujet dans le pays des apprenants.La comparaison faciliterait la compréhension de certains faits.

L'analyse d'une chanson dans la classe FLE est un atout dans l'enseignement d'une langue étrangère. Pourquoi ? Parce qu'elle permet une approche de la langue plus souple et motivante à tous les niveaux. Les étudiants s'intéressent au premier abord à la musique, au rythme, à l'interprétation et puis aux paroles. C'est à ce niveau que la stratégie choisie est essentielle pour déclencher des réactions qui seront à la base de l'enseignement. Le rythme aidant, ils s'intéresseront ensuite aux paroles qui vont donner un sens à la mélodie.

Ces paroles qui abordent certains thèmes, à leur portée ou pas, susciteront l'intérêt des élèves confrontés, par le biais de la chanson, a des réalités lointaines et à des cultures diverses. Ces trois composantes : rythme, paroles, contenu culturel font de la chanson un outil de grande valeur par son caractère sociolinguistique. De plus, c'est un moyen qui motivera les apprenants non seulement à pratiquer la langue d'une manière ludique mais surtout qui leur permettra de débloquer l'expression orale. Faciliter l'expression est le point de départ de la réflexion collective d'abord, personnelle ensuite. La démarche doit introduire le jeu dans la classe afin de créer chez les élèves le plaisir d'apprendre. 
En voici un exemple de son application pour un niveau débutant. Pour un niveau de débutants, l'approche de la culture française avec ses aspects ethnosocioculturels pourrait débuter par la présentation de l'enfance et la société multiculturelle des banlieues avec par exemple la chanson « Je suis » de la chanteuse Amel Bent.

\section{Sensibilisation}

Etant donné le niveau des étudiants, l'enseignant demandera à leur étudiants de faire une recherche préalable, d'apporter des images d'enfants jouant dans des banlieues. Elles seront collées au tableau, décrites et/ou commentées par les élèves. Les élèves caractériseront en 3 mots l'image qu'ils ont de ces enfants. Ils seront notés au tableau.

\section{Phase de découverte de la chanson}

- Écoute de la chanson suivie de questions concernant la musique. De quel type de rythme s'agit-il ? Quelles impressions avez-vous de cette musique ? Quels instruments reconnaissez-vous ? Comment trouvez-vous la voix de la chanteuse?

- Après la deuxième écoute, le professeur posera des questions concernant le contenu. Quel est le thème de la chanson ? Que font les enfants ? A quoi rêve la chanteuse ? Quelles sont les activités évoquéespar le chanteur?

- Cette troisième écoute se fera avec des images correspondant aux paroles. L'enseignant vérifie avec eux si les images correspondent à leur première impression. Il les fera commenter par chaque élève - si l'effectif de la classe le permet - afin d'exploiter au maximum ce support et de pousser les élèves à s'exprimer.

\section{Explications linguistiques et culturelles}

Le professeur fait un petit exercice de révision du vocabulaire connu afin d'éclaircir les doutes possibles. A partir de quelques images le professeur explique le vocabulaire avec la participation active des élèves. Il peut utiliser une « diaporama » de façon à éviter 
de traduire en langue maternelle et à guider les élèves dans la compréhension du vocabulaire. Certains mots seront expliqués avec des synonymes ou mots transparents. Pour vérifier la compréhension, l'enseignant fait faire un exercice rapide d'association. A partir de ces images le professeur fait déduire par les élèves les faits de civilisation en mettant en relief des phrases prononcées par le chanteur.

\section{Acquisition}

Étape très ludique, le professeur fait écouter la chanson avec le karaoké qui associe image / musique / texte. Après les commentaires des élèves, il peut la faire chanter par tout le groupe ou bien faire une sorte de concours avec des volontaires.

\section{Application}

On peut demander aux étudiants de travailler en groupe et de commenter leurs rêves et leurs vœux, ceux des parents, des amis ... Par exemple : Moi, je rêve de, Mon père, lui .... Et puis de décrire comment est la vie au Costa Rica.

Ainsi qu'on peut le constater par la théorie et les exemples donnés, la chanson dans la classe FLE constitue un outil très performant pour aborder et approfondir l'étude des aspects culturels de la société française et, par extension, de la diversité culturelle francophone. Tout d'abord, parce que la culture est indissociable de la langue. En outre, elle facilite également la mise en pratique des quatre compétences, en général et plus particulièrement des compétences communicatives tant au niveau de l'expression que de la compréhension orale d'une manière plus souple. Comme la musique fait partie intégrante de l'univers des apprenants, ils sont conquis par le rythme grâce auquel l'étude linguistico-culturelle est plus motivante et enrichissante. Le choix de la stratégie est extrêmement important car elle doit être dynamique et captiver l'attention des étudiants pour les faire réagir et adhérer aux paroles. 